\title{
Polymer Distributed Bragg Reflectors for Vapor Sensing
}

\author{
Paola Lova, ${ }^{*}{ }^{\dagger}$, Giovanni Manfredi, ${ }^{\dagger}$ Luca Boarino, ${ }^{\S}$ Antonio Comite, ${ }^{\dagger}$ Michele Laus, ${ }^{\prime \prime}$ \\ Maddalena Patrini, ${ }^{\perp}$ Franco Marabelli, ${ }^{\perp}$ Cesare Soci, ${ }^{*}, \#$ and Davide Comoretto ${ }^{* \dagger}$ \\ ${ }^{\dagger}$ Dipartimento di Chimica e Chimica Industriale, Università di Genova, Genova, Italy \\ ${ }^{\ddagger}$ Energy Research Institute at NTU (ERI@AN), Interdisciplinary Graduate School and \# Centre for Disruptive Photonic \\ Technologies, Nanyang Technological University, Singapore \\ ${ }^{\S}$ Nanofacility, National Institute of Metrological Research (INRIM), Torino, Italy \\ "Department of Science and Technology, University of East Piemonte, Vercelli, Italy \\ ${ }^{\perp}$ Department of Physics, University of Pavia, Pavia, Italy
}

\section{Supporting Information}

ABSTRACT: We establish a new strategy to achieve fast and responsive hybrid distributed Bragg reflectors for environmental vapor sensing. We fabricated easily processable zinc oxidepolystyrene nanocomposites to grow high quality multilayers with large gas permeability and dielectric contrast, which enable fast and sensitive detection of vapor analytes. Multilayers fabricated by simple spin-coating of the nanocomposite and cellulose acetate show a 10fold enhancement of the optical response to toluene exposure compared to reference distributed Bragg reflectors built with bare polystyrene.

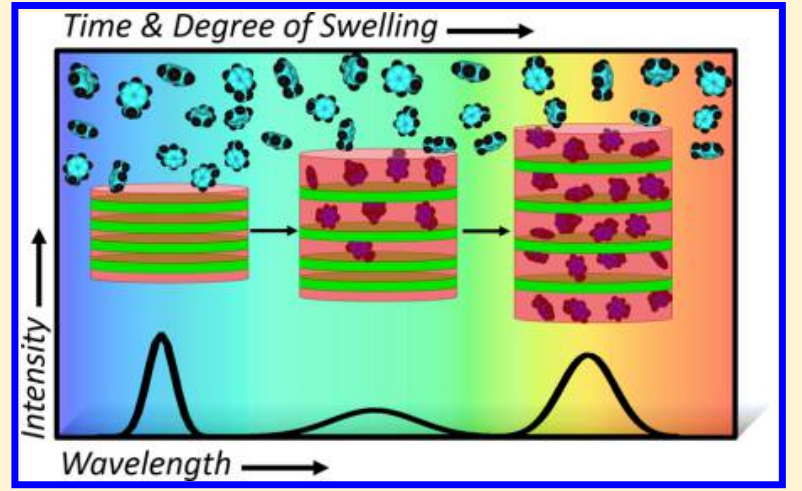

KEYWORDS: $1 D$ photonic crystals, polymer DBR, ZnO nanoparticles, optical nanocomposites, photonic crystal sensors

$\mathrm{D}$ istributed Bragg reflectors (DBRs) are widely used in optoelectronics as antireflective coatings for photovoltaic devices, resonators for lasing cavities, light manipulation in light emitting diodes, and active material in biological and chemical sensors. ${ }^{1-7}$ Thanks to their good mechanical properties, low cost, and ease of fabrication (e.g., by self-assembly, ${ }^{8}$ coextrusion, ${ }^{9}$ or spin-coating ${ }^{3}$ ), polymer-based DBRs are attractive for the production of low-cost, disposable molecule sensors. Molecular detection has already been demonstrated in photonic crystals, where the modification of the optical response is caused by analytes permeation within the structure and, at times, bonding with chemically active additives. ${ }^{10,11}$ The high sensitivity of photonic crystal sensors is related to their photonic band structure, which describes light interaction in a medium with periodic modulation of the refractive index. Forbidden energy regions in the photonic crystal band structure inhibit propagation of isoenergetic light frequencies and generate photonic band gaps whose characteristics strongly depend on the refractive index contrast and its spatial modulation. ${ }^{12,13}$ Penetration of analyte molecules within the crystal modifies the effective refractive index, thus, affecting spectral position, intensity, and width of the photonic band gaps, which can be detected optically.

Despite the widespread use of polymers as building blocks in semi-industrial fabrication processes, ${ }^{9,14}$ so far their use for fabrication of DBR vapor sensors has been hindered by two main issues: first, while highly permeable porous lattices such as liquid crystals, ${ }^{15}$ opals, ${ }^{10,16-22}$ colloidal crystals, ${ }^{11,23,24}$ and porous inorganic ${ }^{25-28}$ and hybrid ${ }^{29,30}$ DBRs are highly sensitive to both liquid and gas analytes, permeability to gas and vapors of polymer DBRs is limited by low porosity of amorphous polymers. This may be overcome by sophisticated optical configurations for the detection of vapors. ${ }^{31-34}$ However, just a few vapor sensitive polymer DBR have been reported so far: in 2003, Convertino et al. showed acetone vapor responsive DBRs made of alternated layers of bare and gold-doped poly(tetrafluoroethylene) fabricated by chemical vapor deposition. ${ }^{35}$ In 2006, Mönch et al. described a multilayer grown by spincoating of monomeric precursors of poly(methyl methacrylate) and polystyrene and in situ polymerization by ultraviolet curing, achieving good sensitivity to acetone and toluene vapors. ${ }^{36}$

The second issue is related to the low dielectric contrast typical of mutually processable polymers. Even though polymer refractive indices in the visible range vary from $n \approx 1.3$ for fluorinated polymers to $n \approx 2.0$ for conjugated systems, coprocessing is restricted by their chemical nature. For instance, coextrusion requires high chemical compatibility which is hardly achievable with fluorinated polymers. ${ }^{9}$ On the other hand, spin-coating requires complete orthogonality between

Received: December 8, 2014

Published: March 2, 2015 
polymers and solvents of alternating layers to prevent dissolution of the underlying films. ${ }^{2}$ Moreover, high absorbance hinders the use of conjugated systems in the visible spectral range and crystalline and semicrystalline polymers scatter light substantially, which is detrimental for optical applications.

In this work we fabricated flexible, free-standing polymer DBRs made of cellulose acetate $(n \approx 1.46)$ and polystyrene $(n$ $\approx 1.57$ ) loaded with high refractive index nanoparticles (Figure 1) and demonstrate their high sensitivity to toluene vapor. In

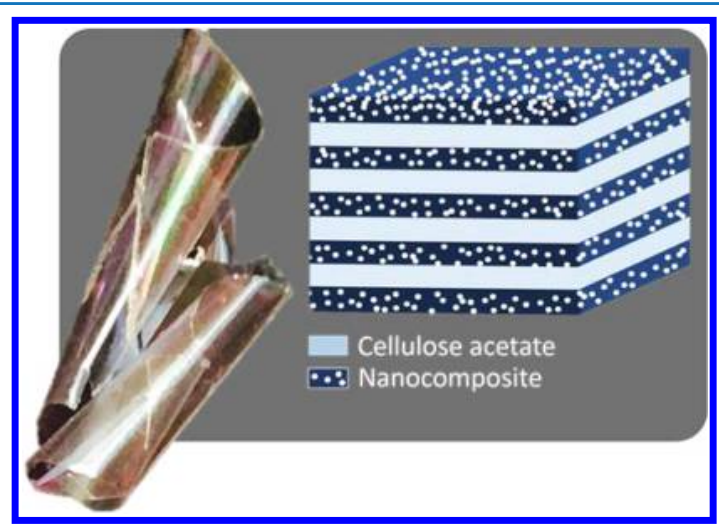

Figure 1. Schematic and photograph of a flexible DBR fabricated with cellulose acetate and polystyrene- $\mathrm{ZnO}$ nanoparticles nanocomposite.

this design, the filler performs a dual function: (i) To introduce free volume in the polystyrene matrix, thus, increasing its vapor permeability. ${ }^{37,38}$ (ii) To increase the matrix effective refractive index (given by the volume-weighted average of the dielectric components) and overall the DBR refractive index contrast. ${ }^{35,39}$ Meanwhile, thanks to the negligible scattering contribution, the nanoparticle filler preserves high transparency of the loaded films.

As a proof of concept, high refractive index $\mathrm{ZnO}$ nanoparticles (NPs, $n \approx 1.98$ ) were synthesized by ultrasoundassisted solvothermal synthesis and properly functionalized to be dispersed in polystyrene. The nanoparticles were then incorporated in a well-studied polymer DBR structure comprising of spun-cast alternating layers of polystyrene and cellulose acetate. ${ }^{2,40}$ Incorporation of optical nanocomposites turns out to be an effective strategy to overcome the major limitations of conventional polymer DBRs: the possibility to increase the dielectric contrast of well-known spin-coatable polymer pairs ${ }^{2,40-42}$ and their permeability at the same time opens up new perspectives for polymer photonic crystal sensing.

\section{RESULTS AND DISCUSSION}

The optical nanocomposite used for DBRs fabrication was prepared by dynamic spin-coating of $\mathrm{ZnO}$ nanoparticles dispersion in polystyrene-toluene solutions. The nanoparticles were synthesized by a solvothermal route (see Methods and Supporting Information for details). Their average size is 14 $\mathrm{nm}$, with a standard deviation of $3.6 \mathrm{~nm}$, as determined by scanning electron microscopy (SEM, see inset of Figure $2 b$ and Supporting Information, Figure S1a). X-ray powder diffraction pattern indicated $13 \mathrm{~nm}$ crystals in Wurtzite phase, ${ }^{43}$ as derived from Debye-Scherrer equation data treatment ${ }^{44}$ and diffraction peak indexing (Supporting Information, Figure S1b).

To achieve good dispersion within the nonpolar matrix, the particles were grafted with a monolayer of dimethyl-(methoxy)

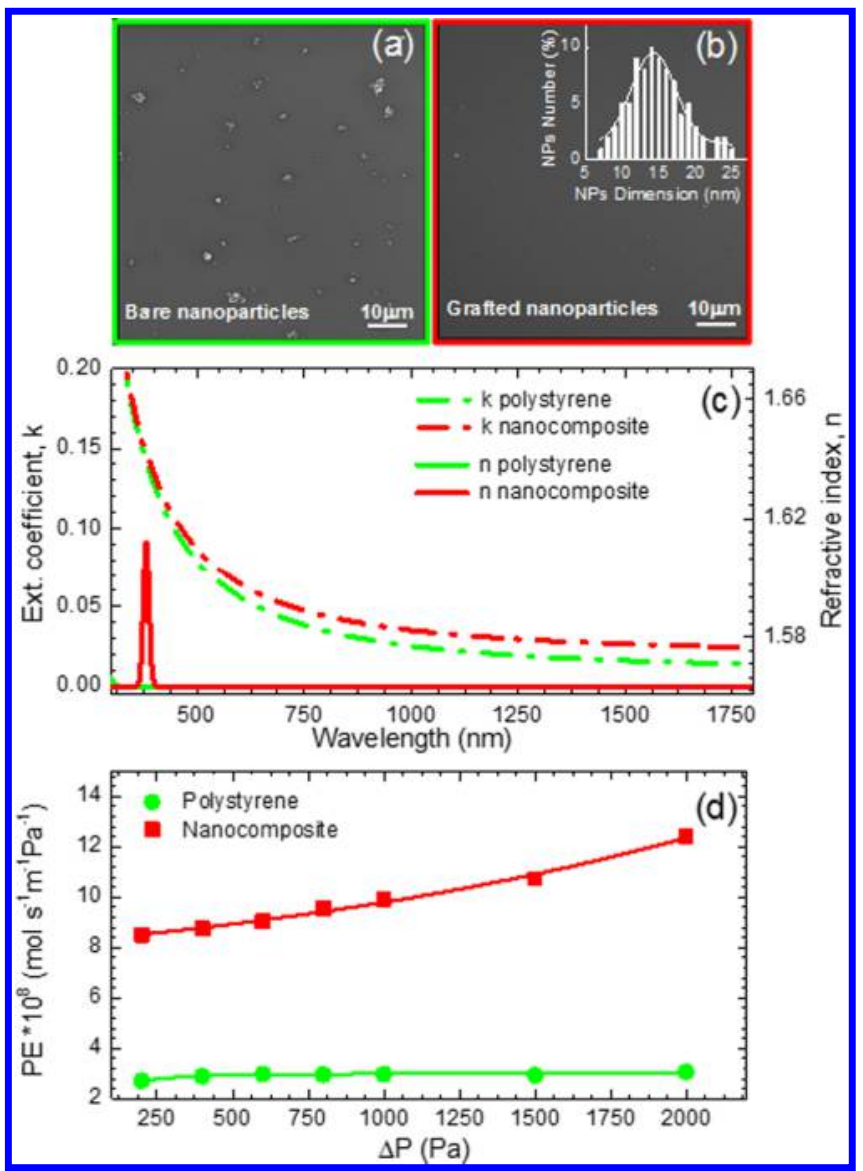

Figure 2. SEM micrographs of nanocomposite thin films loaded with (a) bare $\mathrm{ZnO}$ nanoparticles and (b) nanoparticles grafted with DMMOS. Inset shows the nanoparticles size distribution retrieved from SEM data. (c) Refractive index (dash line) and extinction coefficient (full line) dispersions for bare polystyrene (green) and nanocomposite (red). (d) Permeability to $\mathrm{He}$ gas of polystyrene (green dots) and nanocomposite (red squares) thin films as a function of applied pressure. $\mathrm{ZnO}$ nanoparticles concentration in the nanocomposite is $2.5 \% \mathrm{v} / \mathrm{v}_{\mathrm{PS}}$.

octadecylsilane (DMMOS). Figure $2 \mathrm{a}$ and $\mathrm{b}$ compare the SEM micrographs collected for thin polystyrene film loaded with $2.5 \% \mathrm{v} / \mathrm{v}_{\mathrm{PS}}$ of bare and grafted $\mathrm{ZnO}$ nanoparticles. While several aggregates of few micrometers size are present in the film prepared with bare nanoparticles, the surface of the film containing the grafted particles appears smooth, without signs of aggregation. Negligible light scattering is thus expected from this nanocomposite.

The complex refractive index $(\tilde{n}=n+i k)$ of the thin film nanocomposite derived by spectroscopic ellipsometry confirms the negligible light scattering (Figure 2c). Here, the bare polystyrene extinction coefficient value is zero in the entire spectral range and its refractive index dispersion agrees with literature data $(n=1.581$ at $800 \mathrm{~nm}) .^{2}$ For the nanocomposite, a weak peak due to $\mathrm{ZnO}$ absorption is visible at $380 \mathrm{~nm}$ in the dispersion of $k$. The real part of its refractive index is slightly higher (by less than $0.5 \%$ ) than the one of polystyrene at long wavelengths $(n=1.587$ at $800 \mathrm{~nm})$, while it is nearly identical in the UV. This confirms that a relatively low concentration of high refractive index nanoparticles $\left(2.5 \% \mathrm{v} / \mathrm{v}_{\mathrm{PS}}, 14 \% \mathrm{w} / \mathrm{w}_{\mathrm{PS}}\right)$ does not affect the film transparency. Only at larger nanoparticle concentrations (e.g., 5 and $10 \% \mathrm{v} / \mathrm{v}_{\mathrm{PS}}$ ) light 
scattering becomes significant and prevent the formation of a photonic band gap.

The increase of permeability induced by the loading in the polystyrene matrices was evaluated by pressurized helium permeation tests on free-standing nanocomposite and bare polystyrene thin films (see experimental section). The molar gas flow passing through the sample is related to the permeability (PE) by

$$
\mathrm{PE}=\frac{\mathrm{Ql}}{\Delta P D}
$$

where $Q$ is the He molar flow, $\Delta P$ is the He gas pressure, $l$ and $D$ are the film thickness and the permeation area, respectively (see experimental section for details). Figure $2 \mathrm{c}$ shows that the permeability of bare polystyrene is very low and does not depend on applied pressure. Remarkably, the permeability of the nanocomposite undergoes a 3.5-fold increase and shows strong dependence on applied pressure. This behavior can be attributed to free-volume introduced at the nanoparticlepolystyrene interface, which facilitates gas permeation.

DBR multilayers were fabricated by alternated spin-coating deposition of cellulose acetate and nanocomposite with $\mathrm{ZnO}$ nanoparticles concentration of $2.5 \% \mathrm{v} / \mathrm{v}_{\mathrm{ps}}$. Figure 3 (red line)

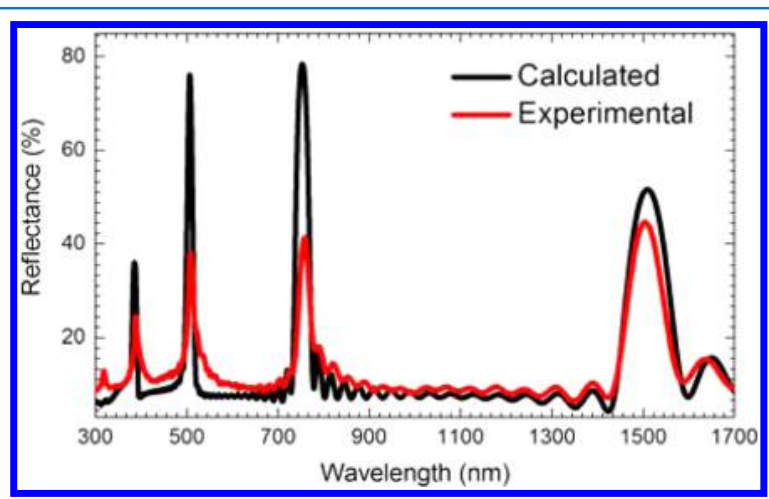

Figure 3. Comparison between experimental (black) and calculated (red) reflectance spectra of a 15 period nanocomposite-cellulose acetate DBR.

shows the reflectance spectrum of a 15 periods DBR with maximum reflectance peaks at $1505\left(\lambda_{\mathrm{PBG}}\right), 758\left(\lambda_{\mathrm{PBG}} / 2\right), 506$ $\left(\lambda_{\mathrm{PBG}} / 3\right), 386\left(\lambda_{\mathrm{PBG}} / 4\right)$, and $317 \mathrm{~nm}\left(\lambda_{\mathrm{PBG}} / 5\right)$. These peak wavelengths correspond to the first order photonic band gap and higher order replicas of the DBR. The baseline of the reflectance spectrum shows an interference pattern typical of high optical quality structures, which arises from the interference of waves partially reflected by the front and back surfaces of the DBR. The DBR optical response was modeled by a homemade software code based on the transfer matrix formalism, using measured refractive indices (Figure $2 b$ and ref 2) and layer thicknesses (Supporting Information, Figure S2) as input. The good agreement between experimental and simulated spectra in Figure 3 confirms the excellent optical quality of the multilayer. Minor discrepancies appear in the UV spectral range, where thickness variations, refractive index precision, and spectrometer sensitivity become critical.

Figure 4 shows the nanocomposite DBR transmittance spectra recorded as a function of incidence angle for $p$ and $s$ light polarization as a contour plot. For both polarizations, the five Bragg peaks shift toward smaller wavelengths increasing the angle of incidence. While in s polarization the width of Bragg

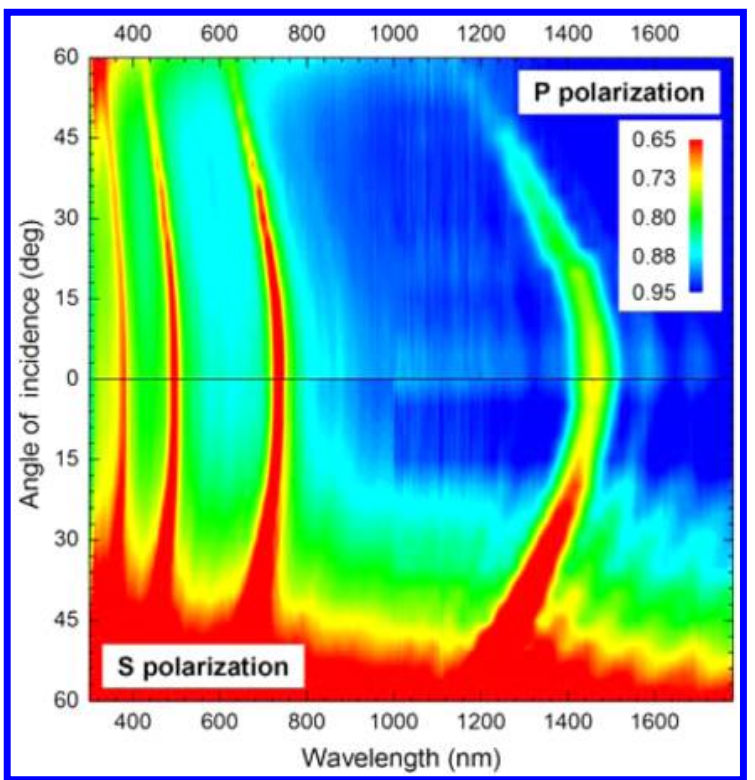

Figure 4. Transmittance spectra contour plot as a function of light incidence angle for $p$ (top panel) and $s$ (bottom panel) light polarization. The color scale represents transmitted intensity.

peaks is nearly independent of the angle of incidence, in $p$ polarization both width and intensity of the peaks decrease toward the Brewster angle. This is consistent with the assignment of photonic band gaps and fully agrees with theoretical predictions (see calculated spectra in Supporting Information, Figure S3). ${ }^{2}$

To evaluate the sensing performance of the nanocomposite $\mathrm{DBR}$, the sample was exposed to toluene vapor in a closed cell and its optical response was compared to that of a control sample fabricated using bare polystyrene with similar film thickness (Figure 5). In the case of the nanocomposite DBR (Figure 5a), the transmittance spectra show a remarkable spectral shift of the photonic bang gap of $\sim 50 \mathrm{~nm}$ after $15 \mathrm{~min}$ of exposure (Figure $5 b$ ), and an $\sim 20 \%$ reduction of the band gap intensity in differential transmittance (Figure 5c). Conversely, no significant changes are observed in either transmittance or differential transmittance spectra of standard polystyrene DBR over time (Figure $6 b^{\prime}$ and $c^{\prime}$ )

The optical response of the DBR vapor sensor at longer exposure times is even richer (Figure 6). After the first $15 \mathrm{~min}$ of exposure to toluene vapor, the transmittance peaks of the nanocomposite DBR undergo nonmonotonic spectral and intensity changes (Figure 6a), while those of the reference polystyrene multilayer show minimal variations in intensity and a only a slight red-shift (Supporting Information, Figure S4). The contour plot in Figure 6a clearly shows transmittance peaks related to the photonic band gap of the DBR (marked by the $*$ symbol), as well as fringes related to the interference of light reflected by the front and back faces of the multilayer structure. The photonic band gap peak red-shifts for the first 15 min (compare to Figure 5a), disappears thereafter, and reappears after $\sim 80 \mathrm{~min}$ at about $1850 \mathrm{~nm}$ (marked by the *' symbol). After $2 \mathrm{~h}$, the spectrum still evolves slowly (data non shown here). When the same measurements are repeated upon diacetone alcohol (cellulose acetate solvent) vapor exposure, no effects are observed in the optical spectra of both polystyrene and nanocomposite multilayers. Toluene and 


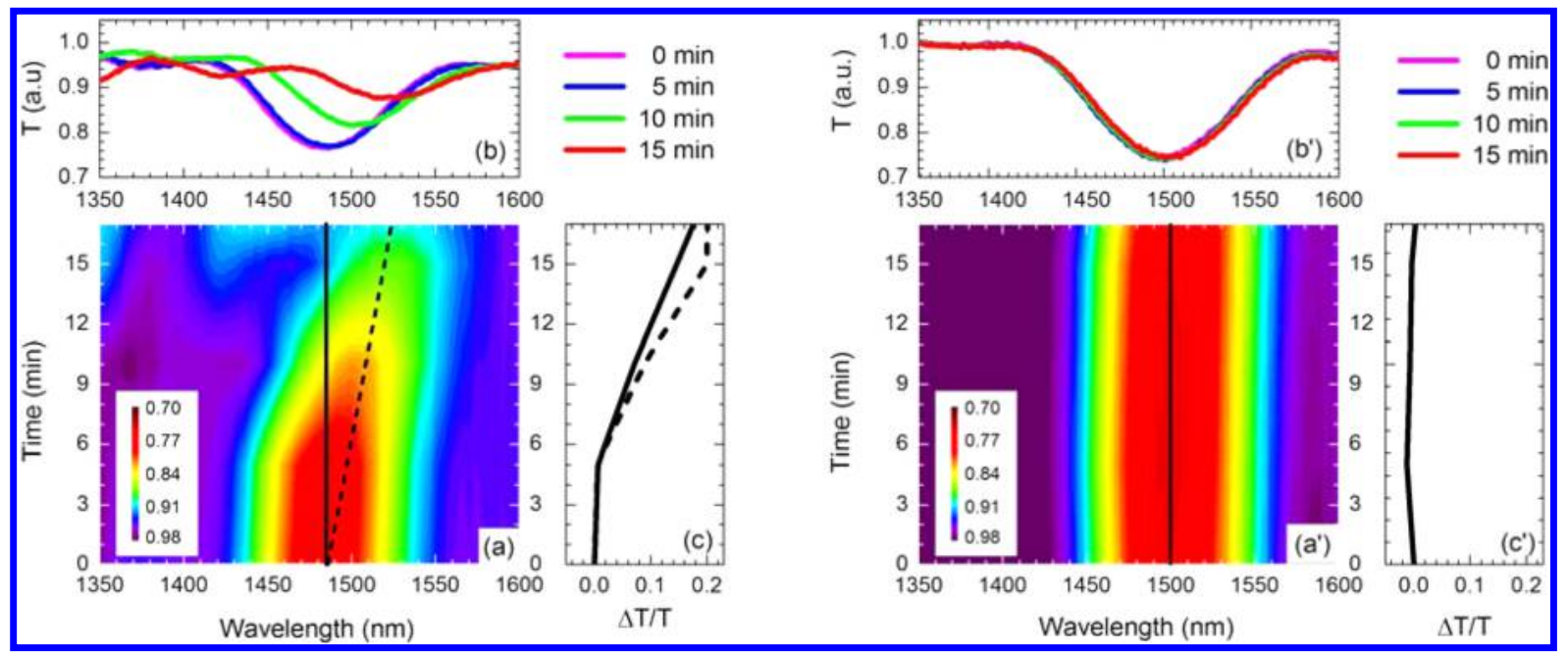

Figure 5. Optical response to toluene vapor exposure of DBR sensors. Nanocomposite DBR on the left and control DBR on the right. (a, $\mathrm{a}^{\prime}$ ) Contour plot of the transmittance spectra collected every $5 \mathrm{~min} ;\left(\mathrm{b}, \mathrm{b}^{\prime}\right)$ Corresponding transmittance spectra; $\left(c, c^{\prime}\right)$ Time-dependent differential transmittance at initial photonic band gap wavelength (solid line in panels a and $a^{\prime}$ ) and minimum of transmission (dashed line in panel a).

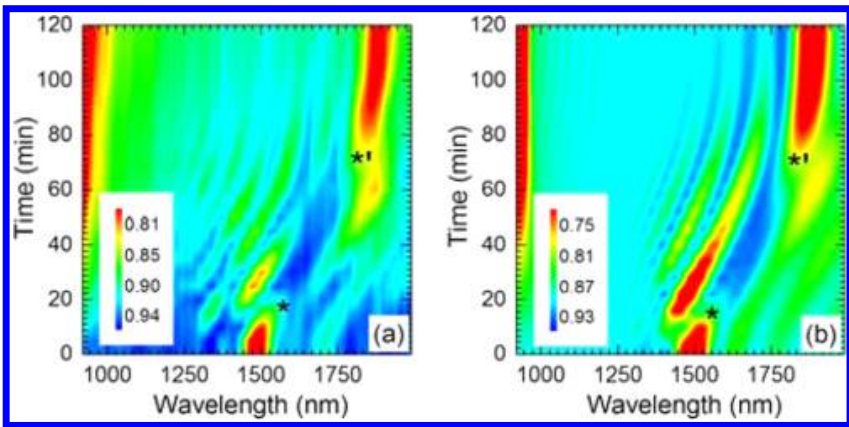

Figure 6. Contour plots of the transmittance spectra collected during the exposure for (a) nanocomposite DBR and (b) control sample. Intensity is represented as a color scale.

diacetone alcohol exposure data are compared in Supporting Information, Figure S4.

The spectral shift of the photonic band gap and its intensity modulation are due to the swelling of the permeable nanocomposite layers induced by toluene molecules that penetrate into the DBR. Indeed, the complex optical response can be reproduced exceptionally well by a fairly simple phenomenological model accounting for the increase of thickness of the nanocomposite layers $\left(D_{\mathrm{NC}}\right)$ upon permeation of solvent vapors from the surface in contact with the toluenerich environment. Let us consider a progressive swelling of the nanocomposite layers over time $(t)$, while the cellulose acetate layer thicknesses $\left(D_{\mathrm{CA}}\right)$ remain constant:

$$
\begin{aligned}
& D_{j, \mathrm{CA}}(t)=D_{j, \mathrm{CA}}(0) \\
& D_{j, \mathrm{NC}}(t)=D_{j, \mathrm{NC}}(0)+\int_{t_{0}}^{t} \frac{\partial D_{j, \mathrm{NC}}(t)}{\partial t} \mathrm{~d} t
\end{aligned}
$$

Under these assumptions, the thickness of each nanocomposite layer $j$ increases over time according to its swelling velocity $\left(\nu_{j}=\partial D_{j, \mathrm{NC}}(t) / \partial t\right)$.

The system of eqs 2 and 3 was solved numerically for discrete time intervals, using the initial layer thicknesses measured by interference microscopy (Supporting Information, Figure S2) as boundary conditions and arbitrarily setting time scale to fit the experimental data.
Note that this model makes two assumptions: (1) The thickness of cellulose acetate layers does not vary with time, and permeation across cellulose acetate occurs instantaneously (one could introduce a time delay due to permeation across cellulose acetate layers, but this would not affect the swelling velocities $\nu_{j}$ and their physical meaning). (2) The variation of the refractive index of the layers upon permeation of solvent vapors is accounted by the variation of the effective optical thickness $\left(\partial D_{j, N C}(t) / \partial t\right)$, which is defined as the product $D_{j} n_{j}$.

The time evolution of each nanocomposite layer thicknesses and swelling velocities derived from the model are reported in Figure 7. The thickness of the first layer increases quickly upon

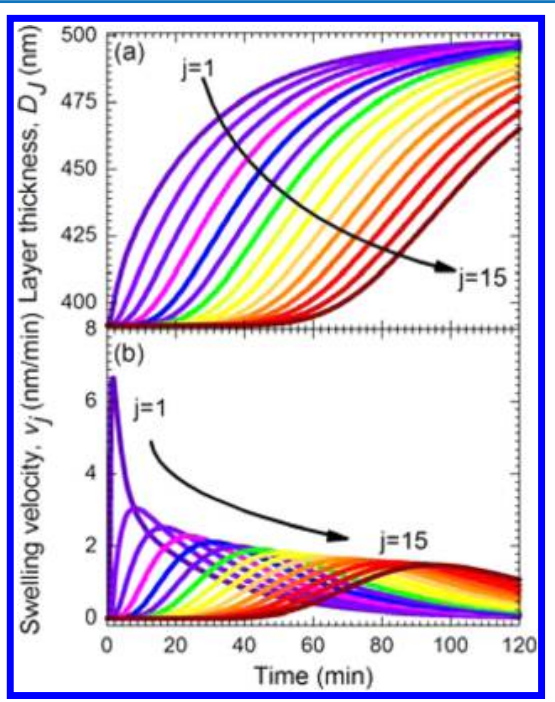

Figure 7. Evolution of the modeling parameters of each nanocomposite layer in the DBR over exposure time: (a) layer thickness and (b) swelling velocity.

toluene exposure; its swelling velocity is very fast initially due to permeation of toluene, and then decreases (Figure $7 b$ ) until the thickness becomes $\sim 25 \%$ larger than the original value (Figure 7a). Toluene permeates progressively into the inner layers of the structures, which then undergo similar swelling process. After about $1 \mathrm{~h}$, the outermost half of the DBR is fully swollen 
while the innermost half is still swelling. Steady-state is reached only after several hours of exposure.

The calculated layer thicknesses were used to model the time evolution of the spectral response of the DBR. The good agreement between experimental (Figure 6a) and calculated (Figure 6b) spectra indicates that the model is able to capture the macroscopic physical changes undergone by the sample, and that progressive swelling of the DBR layers nearest to the surface (close to the toluene rich environment) is indeed responsible for the spectral shift of the DBR peaks. Permeation of toluene vapors across the DBR layers modifies their optical thickness, resulting in a rather complicated but predictable evolution of the transmittance spectra. Within the first $15 \mathrm{~min}$ of exposure, the photonic band gap peak red-shifts due to the thickness increase of the top DBR layers (Figure 6 marked by the symbol $*$ and Figure 7a). Upon toluene penetration in the underlying layers, the diffraction patterns of differently swollen films interfere destructively, and the intensity of the photonic band gap peak is progressively reduced until it cancels after $\sim 20$ min of exposure (Figure 6 between the symbols $*$ and ${ }^{* \prime}$ and Figure $7 \mathrm{a})$. Only after $\sim 80 \mathrm{~min}$ of vapor exposure, when a significant part of DBR layers reaches a stationary swollen condition, the restored optical periodicity results in the appearance of a new, clearly defined band gap (Figure 6 marked by the *' symbol and Figure 7a).We suggest that free volume at the $\mathrm{ZnO}$-polystyrene interface may behave as solvent condensation point, initiating the swelling process, which then propagates to the whole nanocomposite layer.

Figure 8 shows the response time $\left(t_{\mathrm{r}}\right)$ to toluene concentrations ranging from 20 to $120 \mathrm{ppm}$ in air for DBR

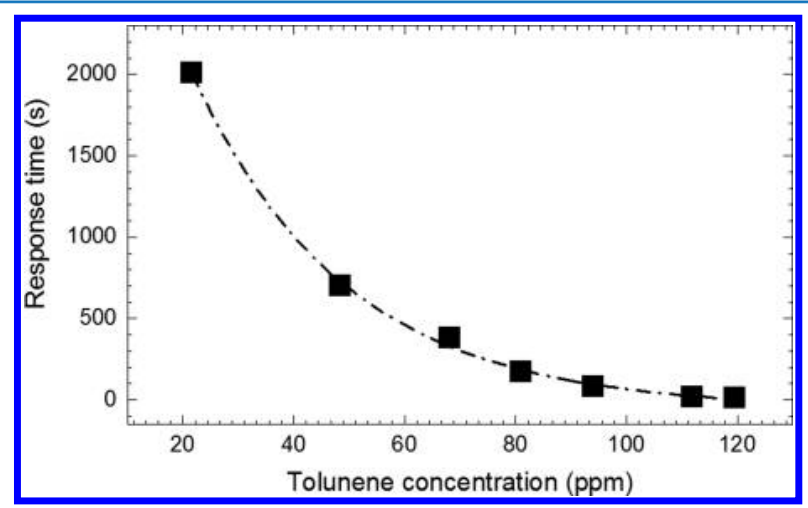

Figure 8. Response time of the nanocomposite sensor to different concentration of toluene vapor for a DBR having photonic band gap at $590 \mathrm{~nm}$.

sensors with photonic band gap at $596 \mathrm{~nm}$ (see Methods and Supporting Information, Figure S5 for details on $t_{\mathrm{r}}$ evaluation). The device is sensitive in the entire concentration range with resolution of $8 \mathrm{ppm}$ (evaluated as the smallest detectable difference in environmental toluene concentration, 120-112 $\mathrm{ppm})$. Note that $t_{\mathrm{r}}$ value decays exponentially upon increases of vapor concentration confirming $8 \mathrm{ppm}$ of resolution in the entire concentration range and allowing to estimate value below $0.5 \mathrm{ppm}$ for concentrations lower than $70 \mathrm{ppm}$.

The measurement time varies from $30 \mathrm{~s}\left(t_{\mathrm{r}}=13 \mathrm{~s}\right)$ for the higher vapor concentration to $60 \mathrm{~min}\left(t_{\mathrm{r}} \sim 33 \mathrm{~min}\right)$ for the lower concentration (see Supporting Information, Figure S5 for details). Our phenomenological prediction and data (Figure 6 and Supporting Information, Figure S4) show that measurement time and, thus, $t_{\mathrm{r}}$ only depend on environmental toluene concentration and DBR layer thickness (i.e., band gap spectral position). Indeed, response time, resolution, and sensitivity can be easily modulated tuning the Bragg peak; devices with photonic band gap in the near-infrared range (thicker nanocomposite layers) will possess higher resolution but slower response than sensors with Bragg peak in the visible range (thinner layers). On the other hand, while near-infrared devices necessitate of a detection system, colored DBR could be used for the qualitative assessment of vapor leakages without any equipment, but just by visual observation.

Concerning the reproducibility, the device optical response is easily restored with less than $2 \%$ error after several cycles of exposure and regeneration by thermal treatment at $50{ }^{\circ} \mathrm{C}$ for 45 s (Supporting Information, Figure S6a,b). However, the time response of the device decreases linearly with the number of cycles performed (Supporting Information, Figure S6c). Analogous behavior was observed for sensors regenerated in environmental conditions (data not shown here). The high reproducibility of the optical response indicates full toluene desorption and restoration of the initial layer thicknesses. However, the shorter response time for exposed devices can be attributed to a modification of the composite morphology, which is likely to be affected from nanoporosity caused by the toluene molecules. Indeed, the sensing process is somehow reversible, but severe thermal treatments are required to achieve full reversibility.

As mentioned earlier, similar effects have been observed in self-assembled electrolytes block copolymer multilayers exposed to solution containing different ions ${ }^{45}$ as well as in highly porous opal structures but, to the best of our knowledge, this is the fastest and most sensitive responsivity to vapors reported for entirely solution-processed DBR prepared by spin-casting. These results are very promising for the fabrication of low cost vapor sensing platforms. However, more comprehensive studies on selectivity and reversibility are necessary to achieve full operating sensing systems.

\section{CONCLUSIONS}

We reported new polymer DBR sensors with high optical responsivity to toluene vapor exposure. Vapor sensitivity was enhanced by increasing the overall DBR permeability with a new polystyrene- $\mathrm{ZnO}$ nanocomposite, which shows permeability $3.5 \times$ higher than bare polystyrene. DBRs displaying up to the fifth diffraction order were fabricated by simple spincoating of alternated layers of cellulose acetate and the new material, which have a slightly higher refractive index than bare polystyrene. The comparison between nanocomposite and polystyrene DBR sensing performance shows that the filler induces an $\sim 10 \times$ faster optical response to toluene vapors exposure leading to fast detection of toluene vapor concentrations ranging between 20 and $120 \mathrm{ppm}$ in air with a resolution below $10 \mathrm{ppm}$. The better response is due to the presence of free-volume induced by the loaded nanoparticles, which allows faster swelling of the polystyrene. The complex dynamic of vapor permeation and swelling of each DBR layer was fully understood in terms of a simple analytical model, which allows predicting the overall DBR optical response up to very long time of exposure to the target vapor. This provides a very robust platform for the design of disposable vapor and gas sensors that are fast and sensitive, for applications in environmental and safety related monitoring such as the detection of hydrocarbons on ocean surface as well as toxic and pollutant gas and vapors in industrial areas. 


\section{METHODS}

Zinc oxide nanoparticles synthesis and functionalization: $\mathrm{ZnO}$ nanoparticles were synthesized via a solvothermal route starting from zinc acetate dihydrate and potassium hydroxide. In a typical process, $0.07 \mathrm{~mol}$ of zinc acetate are dissolved in methanol $(\mathrm{MeOH})$ and heated at $63{ }^{\circ} \mathrm{C}$ under sonication. Then, $0.14 \mathrm{~mol}$ of $\mathrm{KOH}$ are dissolved in the same solvent and slowly added to the first solution. After $3 \mathrm{~h}$ of reaction, the particles are purified by five series of decantation and rinsing with $\mathrm{MeOH}$ and eventually desiccated. ${ }^{46}$ In order to prevent aggregation of $\mathrm{ZnO}$ nanoparticles, which is responsible for light scattering in the nonpolar polystyrene, DMMOS was grafted onto nanoparticles surface. To achieve the grafting, $10 \mathrm{~g}$ of nanoparticle were dispersed in $30 \mathrm{~mL}$ of $\mathrm{MeOH}$ and sonicated. Then, $70 \mathrm{~mL}$ of dichloromethane containing $2.5 \mathrm{~g}$ of DMMOS were added to the dispersion. The overall dispersion dried and desiccated for $2 \mathrm{~h}$ at $40{ }^{\circ} \mathrm{C}$ in a vacuum. The graft reaction between the surface and the adsorbed DMMOS was run at 135 ${ }^{\circ} \mathrm{C}$ under nitrogen flux for $2 \mathrm{~h}$. Nanoparticle growth and grafting reaction mechanism are reported in Supporting Information.

Nanocomposite and DBR preparation: The grafted $\mathrm{ZnO}$ nanoparticles were dispersed into toluene solutions of polystyrene $\left(M_{\mathrm{w}}=200000\right)$ and stirred for more than $36 \mathrm{~h}$. The new colloidal dispersion and bare polymer solutions were used to grow both thin films and DBR by spin-coating. ${ }^{2,3}$ Multilayers were fabricated alternating films of nanocomposite or polystyrene and cellulose acetate $\left(M_{\mathrm{w}}=61000\right)$. The polymer concentrations ranged from 2.8 to $3.5 \%(\mathrm{v} / \mathrm{v})$ and the rotation speed during the deposition was kept at about 6000 $\mathrm{rpm}$. Thin film thicknesses was measured on mono and bilayers made by nanocomposite and cellulose acetate by light interferometry using a GBS smart WLI microscope with a $20 \times$ interference objective.

Materials and DBR characterization: $\mathrm{ZnO}$ nanoparticles and the new nanocomposite thin films were analyzed by SEM using a field emission scanning electron microscope Jeol JSM6700F. The nanoparticles crystal size and structure was identified by XRD. Step scan powder diffraction patterns were collected with a D8 Advance Bruker.

The polymers complex refractive index was determined by spectroscopic ellipsometry with a VASE instrument by J.A. Woollam Co. Inc. and analyzed using the commercial software WVASE32. Incidence angles ranging from $55^{\circ}$ to $75^{\circ}$ have been used.

Thin films permeability were measured with a homemade system schematized in Supporting Information, Figure S7 In this analysis, helium is pressurized and permeated trough freestanding polymer films. The gas flow is measured using a flow meter and related to the sample permeability by eq 1 .

DBR reflectance and transmittance spectra were collected with setups based on optical fiber using an Avantes AvaSpec2048 spectrometer $(200-1150 \mathrm{~nm}$, resolution $1.4 \mathrm{~nm})$ and an Arcoptics FT-interferometer (1000-2600 nm, resolution 8 $\left.\mathrm{cm}^{-1}\right)$. The light source was a combined deuterium-halogen Micropak DH2000BAL.

The sensor response was measured as described in Supporting Information, Figure S5 using a fiber reflection probe for fluids at $22{ }^{\circ} \mathrm{C}$ and $1 \mathrm{~atm}$ in a closed container where a certain amount of toluene was evaporated. The toluene concentration was calibrated via vapor UV-vis spectroscopy. ${ }^{47}$

\section{ASSOCIATED CONTENT}

\section{Supporting Information}

$\mathrm{ZnO}$ nanoparticle SEM characterization and X-ray diffraction pattern. Cellulose acetate and nanocomposite film thicknesses profile, as measured from refraction microscopy. Calculated angle dispersion transmission spectra of nanocomposite DBR. Comparison between DBR optical response to diacetone alcohol and toluene vapors. Evaluation of the sensor time response. Sensing reproducibility. Thin film permeability measurements setup. $\mathrm{ZnO}$ nanoparticles synthesis and grafting reaction mechanisms. This material is available free of charge via the Internet at http://pubs.acs.org.

\section{AUTHOR INFORMATION}

\section{Corresponding Authors}

*E-mail: paola001@e.ntu.edu.sg.

*E-mail: csoci@e.ntu.edu.sg.

*E-mail: davide.comoretto@unige.it.

\section{Notes}

The authors declare no competing financial interest.

\section{ACKNOWLEDGMENTS}

This work is partially supported by Cariplo Foundation Project PHOENICS (No. 2009-2461) and by the Italian Ministry of University and Scientific and Technological Research through the Project 2010XLLNM3 (PRIN 2010-2011). C.S. and P.L. acknowledge support from Nanyang Technological University (M4080538) and the Singapore Ministry of Education (MOE2013-T2-044 and MOE2011-T3-1-005).

\section{REFERENCES}

(1) Calvo, M. E.; Colodrero, S.; Hidalgo, N.; Lozano, G.; LopezLopez, C.; Sanchez-Sobrado, O.; Miguez, H. Porous one dimensional photonic crystals: novel multifunctional materials for environmental and energy applications. Energy Environ. Sci. 2011, 4, 4800-4812.

(2) Frezza, L.; Patrini, M.; Liscidini, M.; Comoretto, D. Directional enhancement of spontaneous emission in polymer flexible microcavities. J. Phys. Chem. C 2011, 115, 19939-19946.

(3) Canazza, G.; Scotognella, F.; Lanzani, G.; De Silvestri, S.; Zavelani-Rossi, M.; Comoretto, D. Lasing from all-polymer microcavities. Laser Phys. Lett. 2014, 11, 035804.

(4) Zheng, G.-G.; Xian, F.-L.; Li, X.-Y. Enhancement of light absorption in thin film silicon solar cells with metallic grating and onedimensional photonic crystals. Chin. Phys. Lett. 2011, 28, 054213.

(5) Hou, L.; Hou, Q.; Mo, Y.; Peng, J.; Cao, Y. All-organic flexible polymer microcavity light-emitting diodes using $3 \mathrm{M}$ reflective multilayer polymer mirrors. Appl. Phys. Lett. 2005, 87, 243504.

(6) Lim, H. S.; Lee, J.-H.; Walish, J. J.; Thomas, E. L. Dynamic swelling of tunable full-color block copolymer photonic gels via counterion exchange. ACS Nano 2012, 6, 8933-8939.

(7) Calvo, M. E.; Sánchez Sobrado, O.; Lozano, G.; Míguez, H. Molding with nanoparticle-based one-dimensional photonic crystals: a route to flexible and transferable Bragg mirrors of high dielectric contrast. J. Mater. Chem. 2009, 19, 3144.

(8) Lee, J.-H.; Koh, C. Y.; Singer, J. P.; Jeon, S.-J.; Maldovan, M.; Stein, O.; Thomas, E. L. 25th Anniversary Article: Ordered polymer structures for the engineering of photons and phonons. Adv. Mater. 2013, 26, 532-569.

(9) Mao, G.; Andrews, J.; Crescimanno, M.; Singer, K. D.; Baer, E.; Hiltner, A.; Song, H.; Shakya, B. Co-extruded mechanically tunable multilayer elastomer laser. Opt. Mater. Express 2011, 1, 108-114.

(10) Ben-Moshe, M.; Alexeev, V. L.; Asher, S. A. Fast responsive crystalline colloidal array photonic crystal glucose sensors. Anal. Chem. 2006, 78, 5149-5157. 
(11) Xu, X.; Goponenko, A. V.; Asher, S. A. Polymerized PolyHEMA photonic crystals: $\mathrm{pH}$ and ethanol sensor materials. J. Am. Chem. Soc. 2008, 130, 3113-3119.

(12) Yablonovitch, E. Inhibited spontaneous emission in solid-state physics and electronics. Phys. Rev. Lett. 1987, 58, 2059-2062.

(13) John, S. Strong localization of photons in certain disordered dielectric superlattices. Phys. Rev. Lett. 1987, 58, 2146-2489.

(14) Song, H.; Singer, K.; Wu, Y.; Zhou, J.; Lott, J.; Andrews, J.; Hiltner, A.; Baer, E.; Weder, C.; Bunch, R.; Lepkowicz, R; Beadie, G. Proc. SPIE 2009, 7467, 74670A-74612.

(15) Stumpel, J. E.; Wouters, C.; Herzer, N.; Ziegler, J.; Broer, D. J.; Bastiaansen, C. W. M.; Schenning, A. P. H. J. An optical sensor for volatile amines based on an inkjet-printed, hydrogen-bonded, cholesteric liquid crystalline film. Adv. Opt. Mater. 2014, 2, 459-464.

(16) Pernice, R.; Adamo, G.; Stivala, S.; Parisi, A.; Busacca, A. C.; Spigolon, D.; Sabatino, M. A.; D’Acquisto, L.; Dispenza, C. Opals infiltrated with a stimuli-responsive hydrogel for ethanol vapor sensing. Opt. Mater. Express 2013, 3, 1820-1833.

(17) Kuo, C.-Y.; Lu, S.-Y.; Chen, S.; Bernards, M.; Jiang, S. Stop band shift based chemical sensing with three-dimensional opal and inverse opal structures. Sens. Actuators, B 2007, 124, 452-458.

(18) Cassagneau, T.; Caruso, F. Conjugated polymer inverse opals for potentiometric biosensing. Adv. Mater. 2002, 14, 1837-1841.

(19) Bonifacio, L. D.; Puzzo, D. P.; Breslav, S.; Willey, B. M.; McGeer, A.; Ozin, G. A. Towards the photonic nose: A novel platform for molecule and bacteria identification. Adv. Mater. 2010, 22, 13511354.

(20) Lin, V. S.-Y.; Motesharei, K.; Dancil, K.-P. S.; Sailor, M. J.; Ghadiri, M. R. A porous silicon-based optical interferometric biosensor. Science 1997, 278, 840-843.

(21) Li, Y. Y.; Cunin, F.; Link, J. R.; Gao, T.; Betts, R. E.; Reiver, S. H.; Chin, V.; Bhatia, S. N.; Sailor, M. J. Polymer replicas of photonic porous silicon for sensing and drug delivery applications. Science 2003, 299, 2045-2047.

(22) Krüger, M.; Marso, M.; Berger, M. G.; Thönissen, M.; Billat, S.; Loo, R.; Reetz, W.; Lüth, H.; Hilbrich, S.; Arens-Fischer, R.; Grosse, P. Color-sensitive photodetector based on porous silicon superlattices. Thin Solid Films 1997, 297, 241-244.

(23) Xie, Z.; Cao, K.; Zhao, Y.; Bai, L.; Gu, H.; Xu, H.; Gu, Z. Z. An optical nose chip based on mesoporous colloidal photonic crystal beads. Adv. Mater. 2014, 26, 2413-2418.

(24) Cui, C.; Liu, Y.; Xu, H.; Li, S.; Zhang, W.; Cui, P.; Huo, F. Selfassembled metal-organic frameworks crystals for chemical vapor sensing. Small 2014, n/a-n/a.

(25) Hinterholzinger, F. M.; Ranft, A.; Feckl, J. M.; Ruhle, B.; Bein, T.; Lotsch, B. V. One-dimensional metal-organic framework photonic crystals used as platforms for vapor sorption. J. Mater. Chem. 2012, 22, 10356-10362.

(26) Lazarova, K.; Awala, H.; Thomas, S.; Vasileva, M.; Mintova, S.; Babeva, T. Vapor responsive one-dimensional photonic crystals from zeolite nanoparticles and metal oxide films for optical sensing. Sensors 2014, 14, 12207-12218.

(27) Exner, A. T.; Pavlichenko, I.; Baierl, D.; Schmidt, M.; Derondeau, G.; Lotsch, B. V.; Lugli, P.; Scarpa, G. A step towards the electrophotonic nose: integrating $1 \mathrm{D}$ photonic crystals with organic light-emitting diodes and photodetectors. Laser Photon. Rev. 2014, 8, 726-733.

(28) Choi, S. Y.; Mamak, M.; von Freymann, G.; Chopra, N.; Ozin, G. A. Mesoporous Bragg stack color tunable sensors. Nano Lett. 2006, 6, 2456-2461.

(29) Karaman, M.; Kooi, S. E.; Gleason, K. K. Vapor deposition of hybrid organic-inorganic dielectric Bragg mirrors having rapid and reversibly tunable optical reflectance. Chem. Mater. 2008, 20, 22622267.

(30) Liu, C.; Yao, C.; Zhu, Y.; Ren, J.; Lan, K.; Peng, H.; Ge, L. Patterned one-dimensional photonic crystals with acidic/alkali vapor responsivity. RSC Adv. 2014, 4, 27281-27285.

(31) Pirotta, S.; Patrini, M.; Liscidini, M.; Galli, M.; Dacarro, G.; Canazza, G.; Guizzetti, G.; Comoretto, D.; Bajoni, D. Strong coupling between excitons in organic semiconductors and Bloch surface waves. Appl. Phys. Lett. 2014, 104, 051111.

(32) Badugu, R.; Nowaczyk, K.; Descrovi, E.; Lakowicz, J. R. Radiative decay engineering 6: Fluorescence on one-dimensional photonic crystals. Anal. Biochem. 2013, 442, 83-96.

(33) Fornasari, L.; Floris, F.; Patrini, M.; Canazza, G.; Guizzetti, G.; Comoretto, D.; Marabelli, F. Fluorescence excitation enhancement by bloch surface wave in all-polymer 1D photonic structure. Appl. Phys. Lett. 2014, 105, 053303.

(34) Clevenson, H.; Desjardins, P.; Gan, X.; Englund, D. High sensitivity gas sensor based on high- $Q$ suspended polymer photonic crystal nanocavity. Appl. Phys. Lett. 2014, 104, 241108.

(35) Convertino, A.; Capobianchi, A.; Valentini, A.; Cirillo, E. N. M. A new approach to organic solvent detection: high-reflectivity bragg reflectors based on a gold nanoparticle/Teflon-like composite material. Adv. Mater. 2003, 15, 1103-1105.

(36) Mönch, W.; Dehnert, J.; Prucker, O.; Rühe, J.; Zappe, H. Tunable Bragg filters based on polymer swelling. Appl. Opt. 2006, 45, 4284-4290.

(37) Ahn, J.; Chung, W.-J.; Pinnau, I.; Guiver, M. D. Polysulfone/ silica nanoparticle mixed-matrix membranes for gas separation. J. Membr. Sci. 2008, 314, 123-133.

(38) Ahn, J.; Chung, W.-J.; Pinnau, I.; Song, J.; Du, N.; Robertson, G. P.; Guiver, M. D. Gas transport behavior of mixed-matrix membranes composed of silica nanoparticles in a polymer of intrinsic microporosity (PIM-1). J. Membr. Sci. 2010, 346, 280-287.

(39) Imai, Y.; Terahara, A.; Hakuta, Y.; Matsui, K.; Hayashi, H.; Ueno, N. Transparent poly(bisphenol A carbonate)-based nanocomposites with high refractive index nanoparticles. Eur. Polym. J. 2009, 45, 630-638.

(40) Unger, K.; Resel, R.; Czibula, C.; Ganser, C.; Teichert, C.; Jakopic, G.; Canazza, G.; Gazzo, S.; Comoretto, D. In 6th International Conference on Transparent Optical Networks (ICTON 2014), Graz, Austria, July 6-10, 2014, Graz University of Technology: Graz, Austria, 2014; Vol. Th.B4.5, pp 1-4.

(41) Álvarez, A. L.; Tito, J.; Vaello, M. B.; Velásquez, P.; Mallavia, R.; Sánchez-López, M. M.; Fernández de Ávila, S. Polymeric multilayers for integration into photonic devices. Thin Solid Films 2003, 433, 277280.

(42) Lova, P.; Manfredi, G.; Boarino, L.; Laus, M.; Urbinati, G.; Losco, T.; Marabelli, F.; Caratto, V.; Ferretti, M.; Castellano, M.; Soci, C.; Comoretto, D. Hybrid $\mathrm{ZnO}$ /polystyrene nanocomposite for allpolymer photonic crystals. Phys. Status Solidi C 2014, n/a-n/a.

(43) Meulenkamp, E. A. Synthesis and growth of $\mathrm{ZnO}$ nanoparticles. J. Phys. Chem. B 1998, 102, 5566-5572.

(44) Klug, H. P.; Alexander, L. E. In X-ray Diffraction Procedures: For Polycrystalline and Amorphous Materials, 2nd ed.; Wiley-VCH: New York, 1974; Vol. 1, pp 992-998.

(45) Kang, Y.; Walish, J. J.; Gorishnyy, T.; Thomas, E. L. Broadwavelength-range chemically tunable block-copolymer photonic gels. Nat. Mater. 2007, 6, 957-960.

(46) Sun, D.; Wong, M.; Sun, L.; Li, Y.; Miyatake, N.; Sue, H.-J. Purification and stabilization of colloidal $\mathrm{ZnO}$ nanoparticles in methanol. J. Sol-Gel Sci. Technol. 2007, 43, 237-243.

(47) Koban, W.; Koch, J. D.; Hanson, R. K.; Schulz, C. Absorption and fluorescence of toluene vapor at elevated temperatures. Phys. Chem. Chem. Phys. 2004, 6, 2940-2945. 